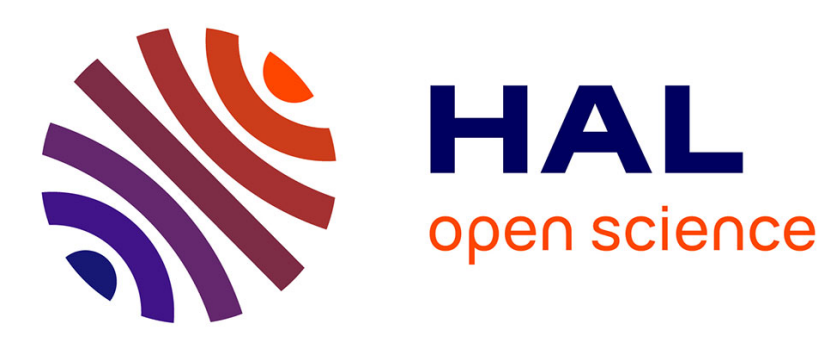

\title{
Enhancing optical-feedback-induced chaotic dynamics in semiconductor ring lasers via optical injection
}

\author{
Nianqiang Li, R. M Nguimdo, A. Locquet, D. Citrin
}

\section{To cite this version:}

Nianqiang Li, R. M Nguimdo, A. Locquet, D. Citrin. Enhancing optical-feedback-induced chaotic dynamics in semiconductor ring lasers via optical injection. Nonlinear Dynamics, 2018, 92 (2), pp.315324. 10.1007/s11071-018-4057-9 . hal-03008323

\section{HAL Id: hal-03008323 https://hal.science/hal-03008323}

Submitted on 16 Nov 2020

HAL is a multi-disciplinary open access archive for the deposit and dissemination of scientific research documents, whether they are published or not. The documents may come from teaching and research institutions in France or abroad, or from public or private research centers.
L'archive ouverte pluridisciplinaire HAL, est destinée au dépôt et à la diffusion de documents scientifiques de niveau recherche, publiés ou non, émanant des établissements d'enseignement et de recherche français ou étrangers, des laboratoires publics ou privés. 


\title{
Enhancing optical-feedback-induced chaotic dynamics in semiconductor ring lasers via optical injection
}

\author{
Nianqiang Li - R. M. Nguimdo - A. Locquet - D. S. Citrin
}

Received: date / Accepted: date

\begin{abstract}
In this paper, we investigate the possibility of using optical injection to efficiently suppress the time-delay (TD) signatures of chaotic signals in a large experimentally accessible parameter range of semiconductor ring lasers (SRLs). We also study how this optical injection can improve the signal bandwidths. The injection signal is obtained from a master SRL with either optical self- or cross- feedback. For optical self-feedback configurations, it is found that the suppression of TD signatures is similar to what has been found in injected Fabry-Perot semiconductor lasers, i.e., narrow range of parameters with respect to the detuning and injection strengths. For cross-feedback configurations, however, the TD signatures can be suppressed in a wide range of parameters meanwhile the bandwidths are significantly improved for the same range of parameters. This is particularly useful for the security in chaos-based communications and also for generating random bits with improved properties.
\end{abstract}

Keywords Chaos · Optical feedback · Optical injection · Time-delay signature - Semiconductor ring laser

\section{N. Li}

School of Computer Science and Electronic Engineering, University of Essex, Wivenhoe Park, Colchester, CO4 3SQ, UK E-mail: wan_103301@163.com

R. M. Nguimdo

Optique Nonlinéaire Théorique, Université Libre de Bruxelles, Campus Plaine, CP 231, 1050 Bruxelles, Belgium

A. Locquet · D. S. Citrin

Department of Electrical and Computer Engineering, Georgia Institute of Technology, Atlanta, GA 30332-0250 USA

UMI 2958 Georgia Tech-CNRS, Georgia Tech Lorraine, 2 rue Marconi, Metz 57070, France

\section{Introduction}

Semiconductor lasers with optical feedback [externalcavity semiconductor lasers (ECLs)] have been the object of intensive research given their potential applications in chaos-based communications, reservoir computing, compressive sensing, and random number generation (see [1-5] and references therein). Recently, however, there has been a marked growth in research on the optimization of their dynamical regimes such as chaos. In particular, the suppression of the time-delay (TD) signature related to the periodicities of the externalcavity modes determined by the external-cavity length has attracted considerable attention (see [2] and references therein). The exposure of a key parameter in ECLs, i.e., feedback TD, is deemed to be a security vulnerability for chaos communications [6]. The existence of noteworthy autocorrelation features around the feedback TD reduces the randomness and increases the post-processing complexity in random number generation [7]. Particularly for chaos-based communications, early works on chaotic lasers with feedback showed that even when the TD signature is concealed in the intensity dynamics $I(t)$ [8], it may in some cases still be inferred by applying the same identifiers to the optical phase dynamics $\phi(t)[9]$. For these reasons, novel approaches for suppressing the TD signature in chaotic sources have been reported [10-19]. Optical injection has been shown as a possible way to generate enhanced chaotic signals, exhibiting suppressed TD signatures, improved chaos bandwidth and dynamical complexity. For example, TD signature suppression in both $I(t)$ and $\phi(t)$ has been achieved by unidirectionally injecting the output of a "master" ECL into a "slave" semiconductor Fabry-Perot laser. But, the injection parameter space region allowing for such signature suppres- 
sions is rather narrow for linewidth-enhancement factors less than 5 [18]. This means that the suppression of TD signatures in large parameter ranges would be hardly achieved in optically injected Fabry-Perot lasers because linewidth-enhancement factor is typically less than or equal to 5 in lasers. It is therefore of interest to explore whether the suppression of the TD signatures can be obtained in other lasers under the optical injection in a large parameter space regions even for moderate linewidth-enhancement factors (i.e., $\leq 5$ ).

In this paper, we choose to carry out such a study using semiconductor ring lasers (SRLs). Our choice is motivated by the fact that, among the different lasers, SRLs are characterized by a cavity with a circular geometry, and thus can support two counter-propagating modes: a clockwise (CW) and a counter-clockwise mode (CCW) [20,21]. Such unique features can allow the implementation of different feedback configurations: a single SRL can be used to implement a self-feedback configuration (a part of the output signal from one direction is injected back to the same direction) [22] or a cross-feedback configuration (a part of the output signal from one direction is injected back to the opposite direction) [23]. With cross-feedback configuration, it has been proven that it is possible to generate chaotic signals with extremely weak TD signatures both in the intensity and the phase dynamics [23]. However, this simultaneous TD concealment both in the intensity and phase dynamics is possible only either for relatively large values of linewidth enhancement factor (i.e., $\geq 5$ while the typical value is $\approx 3.5$ ) or for self-and cross-saturation values which are about 10 times less than the typical values. In addition, chaos bandwidth is typically low in SRLs as compared to Fabry-Perot lasers. These constraints would limit the use of a single SRL with feedback for achieving high speed applications. To improve this performance, we numerically investigate here whether a "slave" SRL (S-SRL) optically injected by a "master" SRL (M-SRL) under optical self- or cross-feedback can generate chaotic dynamics with improved properties. In particular, we investigate the ability of this system for simultaneously achieving TD concealment and improved bandwidths in a large range of experimentally accessible parameters even under moderate linewidth enhancement factors. We show the results of the TD signatures both for the intensity and phase dynamics when the M-SRL is under either self-feedback (SOF) or cross-feedback (COF). To illustrate the benefit of enhanced dynamics features on a specific application, we show the randomness features of two bitstreams generated one from a master dynamics and another from the slave response to this master's dynamics for substantial slave SRL parameters where the TD concealment and improved bandwidths are obtained.

The paper is organized as follows. The theoretical model for an optically injected SRL is presented in Section 2 . Section 3 presents the results for the suppression of TD signatures, and compares the cases of M-SRL under SOF and COF. In Section 4, the enhancement of chaos bandwidth is studied briefly. In Section 5, we apply the time series with and without TD signatures to random bit generation, and discuss their performance. And finally, a basic conclusion is given in Section 6 .

\section{Numerical model}

The system consists of a "slave" SRL optically injected by a "master" SRL with either SOF [Fig.1(a)] or COF [Fig.1(b)] feedback. For each configuration, a part of the output signal of M-SRL directional mode is injected into the same directional mode of the S-SRL, i.e., $\mathrm{CW}$-to-CW and CCW-to-CCW. Figure 2 shows the full scheme under investigation.

The dynamics of the system can be obtained by simulating the two-mode rate equation model assuming that SRLs operate in a single longitudinal mode [24],

$$
\begin{aligned}
\dot{E}_{1 a}= & \kappa(1+i \alpha)\left[g_{1 a} N_{a}-1\right] E_{1 a}-k(1-\delta k) e^{i \phi} E_{2 a} \\
& +\eta_{1} \mathcal{F}_{1}(t), \\
\dot{E}_{2 a}= & \kappa(1+i \alpha)\left[g_{2 a} N_{a}-1\right] E_{2 a}-k(1+\delta k) e^{i \phi} E_{1 a} \\
& +\eta_{2} \mathcal{F}_{2}(t), \\
\dot{E}_{1 b}= & \kappa(1+i \alpha)\left[g_{1 b} N_{b}-1\right] E_{1 b}-k(1-\delta k) e^{i \phi} E_{2 b} \\
& +\gamma E_{1 a}\left(t-\tau_{i n j}\right) e^{-i \omega_{a} \tau_{i n j}+i 2 \pi \Delta f t}, \\
\dot{E}_{2 b}= & \kappa(1+i \alpha)\left[g_{2 b} N_{b}-1\right] E_{2 b}-k(1+\delta k) e^{i \phi} E_{1 b} \\
& +\gamma E_{2 a}\left(t-\tau_{i n j}\right) e^{-i \omega_{a} \tau_{i n j}+i 2 \pi \Delta f t}, \\
\dot{N}_{a, b}= & \gamma_{N}\left[\mu-N_{a, b}-g_{1 a, 1 b} N_{a, b}\left|E_{1 a, 1 b}\right|^{2}\right. \\
& \left.-g_{2 a, 2 b} N_{a, b}\left|E_{2 a, 2 b}\right|^{2}\right] .
\end{aligned}
$$

where the subscript $a, b$ stands for the M-SRL (S-SRL), $E_{1}$ and $E_{2}$ are the slowly varying complex envelopes of $\mathrm{CW}$ and CCW modes, and $N$ is the carrier population inversion. The last terms in Eqs.(1) and (2) account for the feedback. For SOF configuration, $\mathcal{F}_{1}(t)=E_{1}(t-$ $\left.\tau_{2}\right) e^{i \theta}$ and $\mathcal{F}_{2}(t)=E_{2}\left(t-\tau_{1}\right) e^{i \theta}$. For COF configuration, $\mathcal{F}_{1}(t)=E_{2}\left(t-\tau_{2}\right) e^{i \theta}$ and $\mathcal{F}_{2}(t)=E_{1}\left(t-\tau_{1}\right) e^{i \theta} \cdot \eta_{1}$ and $\eta_{2}$ are feedback rates, $\tau_{1}$ and $\tau_{2}$ are delay times, and $\theta$ is the constant feedback phase. Note that $\eta=\eta_{1}=\eta_{2}$ and $\tau=\tau_{1}=\tau_{2}$ are assumed for the case of SOF. The last terms in Eqs.(3) and (4) account for the optical injection, $\omega_{a}$ being the solitary laser frequency of the MSRL, $\gamma$ the injection rate, $\tau_{i n j}$ the coupling delay time and $\Delta f$ frequency detuning. $g_{1}=1-s\left|E_{1}\right|^{2}-c\left|E_{2}\right|^{2}$ 
(a) SOF

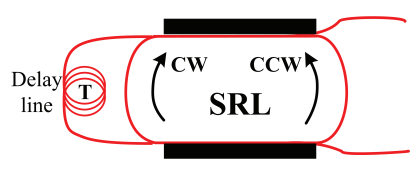

(b) $\mathrm{COF}$

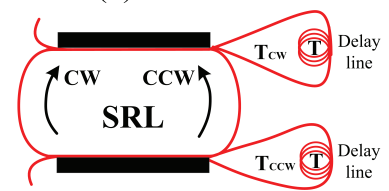

Fig. 1 Schematic of the master SRL with (a) SOF and (b) COF. CW: clockwise mode, CCW: counter-clockwise mode. The slave SRL is similar to the master SRL but does not have feedback.

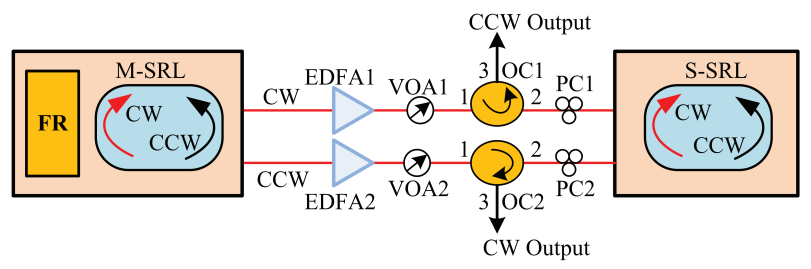

Fig. 2 Schematic of an optically injected SRL. SRL, semiconductor ring laser; M-SRL, master SRL; S-SRL, slave SRL; CW, clockwise; CCW, counterclockwise; EDFA, erbiumdoped fiber amplifier; VOA,variable optical attenuator; OC, optical circulator; PC, polarization controller; FR, optical fiber reflector.

and $g_{2}=1-s\left|E_{2}\right|^{2}-c\left|E_{1}\right|^{2}$ are the differential gains in which $s$ and $c$ are phenomenological self- and crosssaturation coefficients, $\mu$ is the renormalized injection current $(\mu=0$ at transparency and $\mu=1$ at lasing threshold), $\kappa$ is the field decay rate, $\gamma_{N}$ is the carrier decay rate, and $\alpha$ is the linewidth enhancement factor. The linear coupling between the two counterpropagating modes is added through the backscattering rate $k e^{i \phi}$ modeled by an amplitude $k$ and a phase shift $\phi$. The intrinsic backscattering is, in general, asymmetric due to unavoidable imperfections introduced during device fabrication [25]. Therefore, an asymmetry $\delta k$ is added in the backscattering amplitude, but this asymmetry does not have significant effect on the TD signatures.

In our simulations, we assume identical internal parameters for the two SRLs and we neglect spontaneousemission noise: $\alpha=3.5, \mu=2.5, s=0.005, c=0.01$, $\kappa=100 \mathrm{~ns}^{-1}, \gamma_{N}=0.2 \mathrm{~ns}^{-1}, k=0.44 \mathrm{~ns}^{-1}, \delta k=0.2$, $\phi=\theta=1.5, \eta_{1}=5 \mathrm{~ns}^{-1}, \eta_{2}=6 \mathrm{~ns}^{-1}, \eta=2.5 \mathrm{~ns}^{-1}$, $\tau_{1}=5 \mathrm{~ns}, \tau_{2}=7 \mathrm{~ns}, \tau=6 \mathrm{~ns}, \omega_{a}=2.22 \times 10^{15}$ $\mathrm{rad} \cdot \mathrm{s}^{-1}$, and $\tau_{i n j}=0$ ns. These parameters are typical of laser diodes consisting of a cavity with a circular geometry $[22,23]$. Our control parameters are therefore the injection strength and the detuning $\Delta f$. With these parameters, the relaxation period of the free-running SRL is $\tau_{R O}=2 \pi / \sqrt{2(\mu-1) \gamma_{N} \kappa}=0.81 \mathrm{~ns}$, which is much smaller than the feedback delay times (i.e., longcavity regime). Note that the feedback strength $\eta$ has been chosen such that all the time-delays can be readily retrieved by computing the known delay identifiers from the M-SRL signals. Thus, for different values of $\gamma$ and $\Delta f$, we will systematically investigate the conditions under which the S-SRL suppresses the TD signatures identifiable on the M-SRL signals. While other TD identifiers exist, we will use the autocorrelation function (ACF) which has proven to be efficient $[8,13]$. It will be computed from the intensity time series $I(t)$ or from the phase time series $\phi(t)$, and the peaks around the delay times and their multiples will be identified as TD signatures when they can be clearly distinguished from the background noise. The ACF measures how well a time series $x(t)$ matches in the linear sense its time-shifted by $\Delta t$ version $x_{s}(t)[9]$. It is defined as

$$
\begin{aligned}
& C(\Delta t)= \\
& \frac{\left\langle\left[x_{a}(t)-\left\langle x_{a}(t)\right\rangle\right]\left[x_{b, s}(t)-\left\langle x_{b, s}(t)\right\rangle\right]\right\rangle}{\sqrt{\left\langle\left[x_{a}(t)-\left\langle x_{a}(t)\right\rangle\right]^{2}\right\rangle\left\langle\left[x_{b, s}(t)-\left\langle x_{b, s}(t)\right\rangle\right]^{2}\right\rangle}},
\end{aligned}
$$

with $a=b, x_{s}(t)=x(t+\Delta t)$, and $<\cdot>$ the time (i.e., $t$ ) average. To quantify the correlation between two different time series (i.e., $a \neq b$ ), we define the cross-correlation coefficient $\rho(\Delta t)$ in the same way. The value of the correlation coefficient lies in the range $-1 \leq$ $\rho(\Delta t) \leq 1$ with higher value of $|\rho(\Delta t)|$ meaning better synchrony.

\section{Numerical results}

The rate equations have been integrated using a standard fourth-order Runge-Kutta method with the fixed time step of 1 ps. We only consider the cases for which the M-SRL yields chaotic dynamics in both modes.

\subsection{M-SRL under self-feedback}

Figure 3(a1) shows a typical temporal profile of the intensity $I(t)$ for CW (blue) and CCW (red) modes of the M-SRL under SOF while Fig. 3(a2) shows their associated optical spectra. The broadband featureless nature of both indicates the master laser operates in a chaotic regime. Figure 3(b1) and 3(b2) display the ACF computed from $I(t)$ and $\phi(t)$, respectively. As expected, pronounced peaks are observed close to the TD position and its multiples, i.e., $\tau$ and $T=2 \tau$, in the ACF of $I(t)$ and $\phi(t)$. In particular, it can be noted that the peaksize at $\tau$ is higher than that at $T$. This means that $\tau$ is the time-delay which dominates the dynamics of each mode. Also, only a moderate feedback level of $\eta=2.5$ $\mathrm{ns}^{-1}$ has been considered. More obvious TD signature is observed for larger values of $\eta$. So, the TD signatures can be always inferred both from the intensity and the phase dynamics of M-SRL under SOF. 

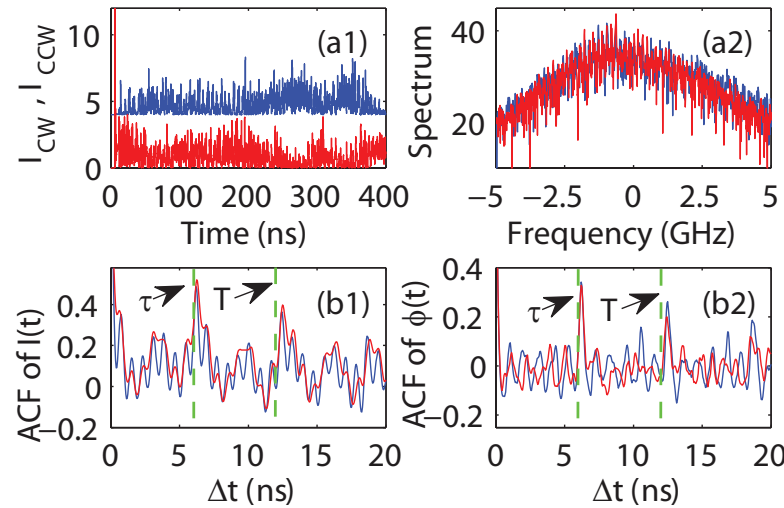

Fig. 3 (a1) CW and CCW intensities in M-SRL under SOF, (a2) their associated optical spectra, (b1) ACF computed from $I(t)$, and (b2) ACF computed from $\phi(t)$. Red: CCW; blue: CW. Vertical dashed lines indicate the different delays. The time traces for CW mode are vertically shifted for better visual clarity, whereas the spectra are overlapped.
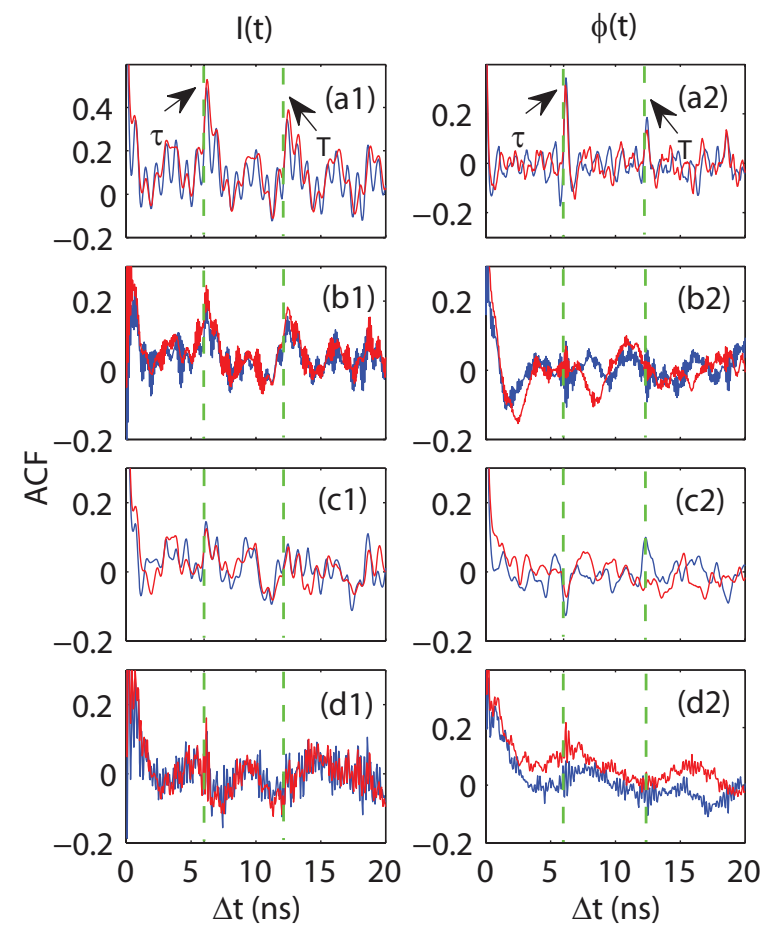

Fig. 4 ACF computed from $I(t)$ (left) and $\phi(t)$ (right) in the S-SRL for (a) $\Delta f=0 \mathrm{GHz}$ and $\gamma=35 \mathrm{~ns}^{-1}$, (b) $\Delta f=10$ $\mathrm{GHz}$ and $\gamma=35 \mathrm{~ns}^{-1}$, (c) $\Delta f=20.4 \mathrm{GHz}$ and $\gamma=84 \mathrm{~ns}^{-1}$, and (d) $\Delta f=25 \mathrm{GHz}$ and $\gamma=85 \mathrm{~ns}^{-1}$. Red: CCW; blue: CW. Vertical dashed lines indicate the different delays.

When the master is unidirectionally coupled to the S-SRL, the latter's response depends on its parameters. Figure 4 shows examples of TD signatures of the S-SRL for different injection conditions. In (a), the S-SRL is locked to the chaotic optical injection from the M-SRL such that the outputs of the two lasers are highly cor-

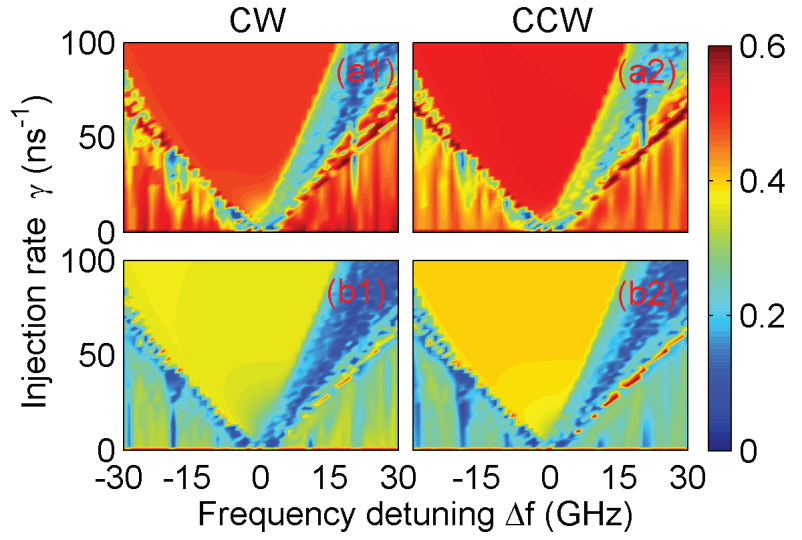

Fig. 5 ACF Peaksize computed from $I(t)$ of CW (left) and CCW (right) as functions of $\gamma$ and $\Delta f$ in the S-SRL for (a1) and (a2) $\tau$, (b1) and (b2) $T$.

related. In such a case, the S-SRL shows similar TD signatures as the M-SRL. However, for substantial values of $\gamma$ and $\Delta f$, significant reduction of all the TD signatures is found [see Figs. 4(b)-(d)].

In order to identify the parameter range for which the S-SRL can successfully suppress the TD signatures, we extract the peaksize in the autocorrelation i.e., the maximum autocorrelation peak (absolute value) in time windows around time-delay $x, W(x)=[x-0.02 x, x+$ $0.02 x]$, where $x=\{\tau, T\}$. The ratio 0.02 was chosen such that the considered range is large enough to include its own delay peak and should also remove interference from the ACF peak of other delays in the system. Figure 5 displays the results for the peaksize computed from $I(t)$ in $(\Delta f, \gamma)$-plane for $\tau(\mathrm{a} 1, \mathrm{a} 2)$ and $T$ (b1, b2) considering the $\mathrm{CW}$ and CCW dynamics of the S-SRL. As in optically injected edge-emitting lasers (EELs) [18], it is seen that a very narrow range of $\gamma$ and, mostly positive, $\Delta f$ values (dark blue) ensures weak TD signatures both in $\mathrm{CW}$ and $\mathrm{CCW}$ modes.

\subsection{M-SRL under cross-feedback}

Figure 6(a1) displays some typical temporal profiles of $I(t)$ for CW (blue) and CCW (red) modes of the M-SRL under COF, and Fig. 6(a2) shows their associated optical spectra. Figures 6(b1) and 6(b2) display the ACF computed from $I(t)$ and $\phi(t)$, respectively. It is seen that only the delay signature related to $T$ can be inferred from the ACF from $\phi(t)$. However, pronounced peaks are observed close to the TD positions and their multiples, i.e., $\tau_{1}, \tau_{2}$, and $T \approx \tau_{1}+\tau_{2}$ when the ACF is computed from $I(t)$, as also shown in [23].

Figure 7 displays several distinct examples of the TD signatures of the S-SRL under various injection 

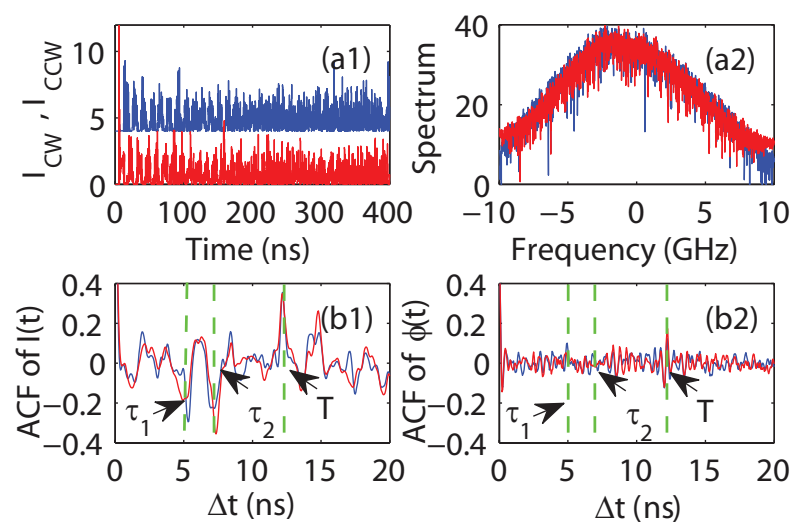

Fig. 6 (a1) CW and CCW intensities in M-SRL under COF, (a2) their associated optical spectra, (b1) ACF computed from $I(t)$, and (b2) ACF computed from $\phi(t)$. Red: CCW; blue: CW. Vertical dashed lines indicate the different delays. The time traces for CW mode are vertically shifted for better visual clarity, whereas the spectra are overlapped.
I(t)
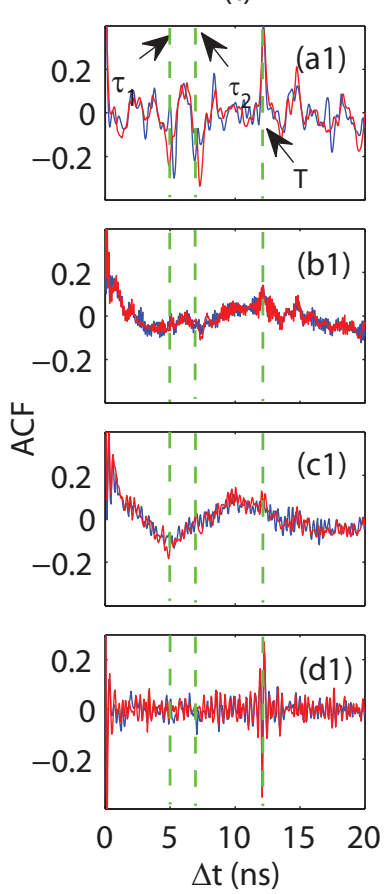

$\phi(\mathrm{t})$
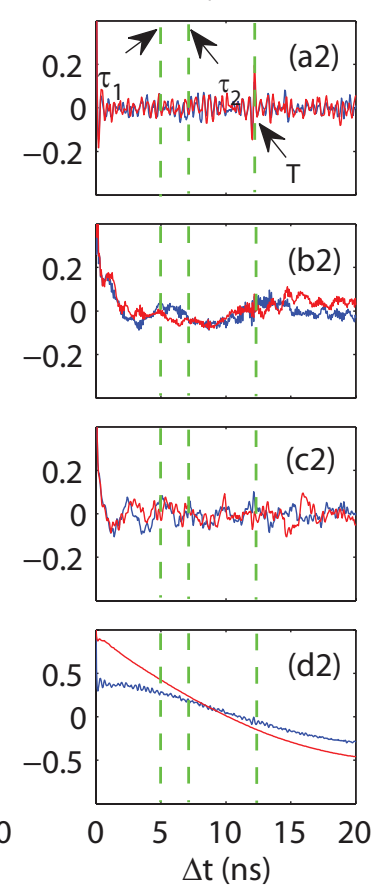

Fig. 7 ACF computed from $I(t)$ (left) and $\phi(t)$ (right) in the S-SRL for (a) $\Delta f=0 \mathrm{GHz}$ and $\gamma=20 \mathrm{~ns}^{-1}$, (b) $\Delta f=5$ $\mathrm{GHz}$ and $\gamma=10 \mathrm{~ns}^{-1}$, (c) $\Delta f=15 \mathrm{GHz}$ and $\gamma=48 \mathrm{~ns}^{-1}$, and (d) $\Delta f=-15 \mathrm{GHz}$ and $\gamma=15 \mathrm{~ns}^{-1}$. Red: CCW; blue: CW. Vertical dashed lines indicate the different delays.

conditions. Three cases can be distinguished: i) Timedelays can be readily retrieved as in the master when the S-SRL is locked to the M-SRL [Figs. 7(a1) and (a2)]; ii) all TD signatures are strongly attenuated as a result of the interaction between the optical injection and the relaxation oscillation of the slave laser [Figs. 7(b1),
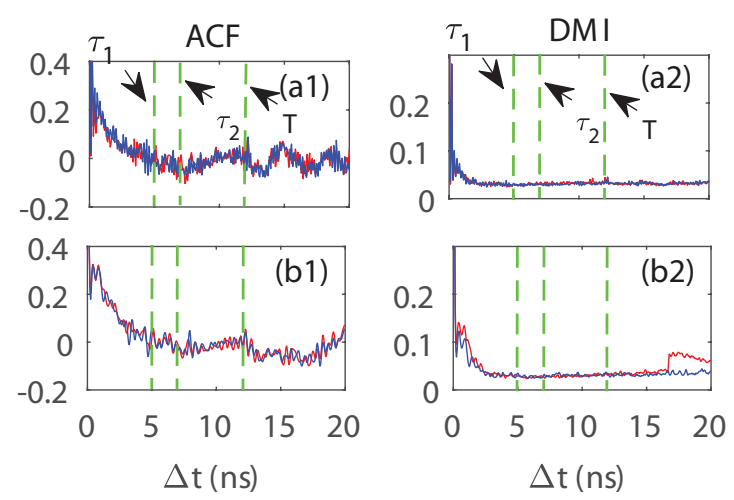

Fig. $8 \mathrm{ACF}$ (left) and DMI (right) computed from $I(t)$ in the S-SRL for (a) $\Delta f=17 \mathrm{GHz}$ and $\gamma=52 \mathrm{~ns}^{-1}$, and (b) $\Delta f=20 \mathrm{GHz}$ and $\gamma=57 \mathrm{~ns}^{-1}$. Red: CCW; blue: CW. Vertical dashed lines indicate the different delays.

(b2), (c1) and (c2)]; iii) only the TD corresponding to the total delay $T$ and its multiples can be identified from the ACF of $I(t)$ or $\phi(t)$ [Figs. $7(\mathrm{~d} 1)$ and $(\mathrm{d} 2)$ ], while the signatures of the feedback delays $\left(\tau_{1}\right.$ and $\left.\tau_{2}\right)$ are strongly suppressed both in $I(t)$ and $\phi(t)$. From the time series (not shown) of the intensity and phase dynamics, however, we have found that the slave dynamics becomes very weakly chaotic for the values of $\Delta f$ and $\gamma$ used in Fig. $7($ d) [i.e., $\Delta f=-15 \mathrm{GHz}$ and $\gamma=15$ $\left.\mathrm{ns}^{-1}\right]$. This may explain why the TD corresponding to the total delay $T$ can be readily inferred in this case.

To further confirm the strong suppression of all TD signatures involved, we employ another TD identifier, delayed mutual information (DMI), which is a statistic measure based on information theory, measuring the nonlinear dependence of two variables; see $[8,9]$ for the definition. We consider two additional sets of the injection parameter and present the results for $\mathrm{ACF}$ and DMI computed from $I(t)$ in the S-SRL in Fig. 8. (The results for $\phi(t)$ are not shown, since the phase variable always embodies better concealment of TD signatures; see Fig. 7.) A comparison between these ACF and DMI plots shows excellent agreement between both identifiers as expected, indicating that, on the one hand, all TD signatures are effectively suppressed, and on the other hand, we have ample reason to just use the single tool, ACF, for detecting TD signatures throughout this study.

Figure 9 shows a full scan in the $(\gamma, \Delta f)$-plane of how the peaksizes around the time-delays $\tau_{1}, \tau_{2}$, and $T$ evolve. Here the peaksizes are retrieved in time windows around a delay time $x, W(x)=[x-0.02 x, x+0.02 x]$, where $x=\left\{\tau_{1}, \tau_{2}, T\right\}$. Again, only the results of the ACF from $I(t)$ are displayed here because Fig. 7 has shown that the ACF computed from $I(t)$ is more suitable to identify the time-delays for COF configuration. It turns out that $\mathrm{CW}$ and $\mathrm{CCW}$ modes have similar 


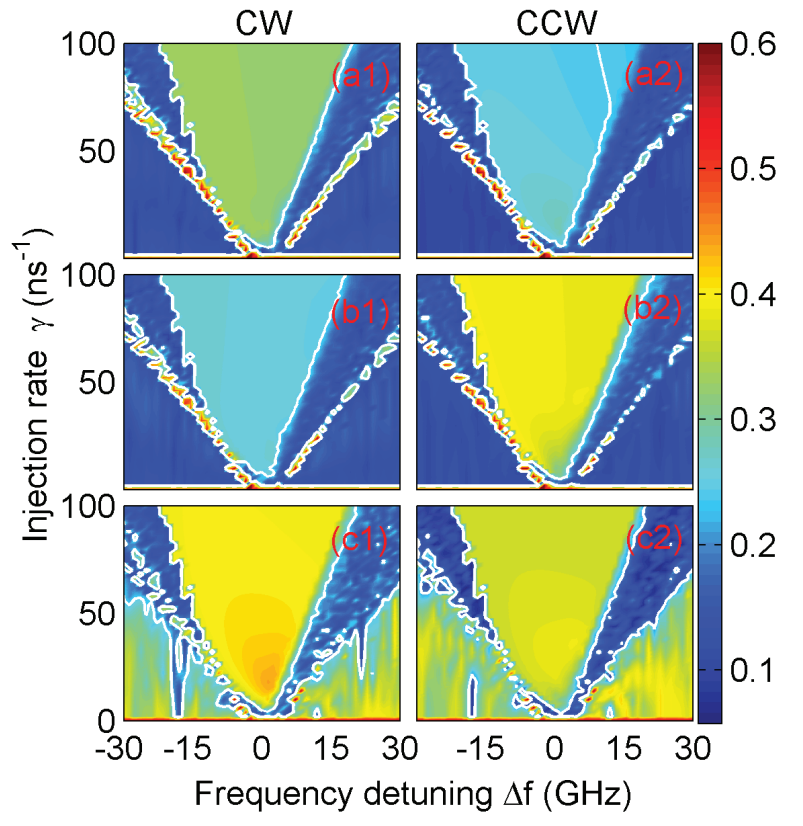

Fig. 9 ACF Peaksize computed from $I(t)$ of CW (left) and CCW (right) as functions of $\gamma$ and $\Delta f$ in the S-SRL for (a1) and (a2) $\tau_{1},(\mathrm{~b} 1)$ and (b2) $\tau_{2},(\mathrm{c} 1)$ and (c2) $T$.

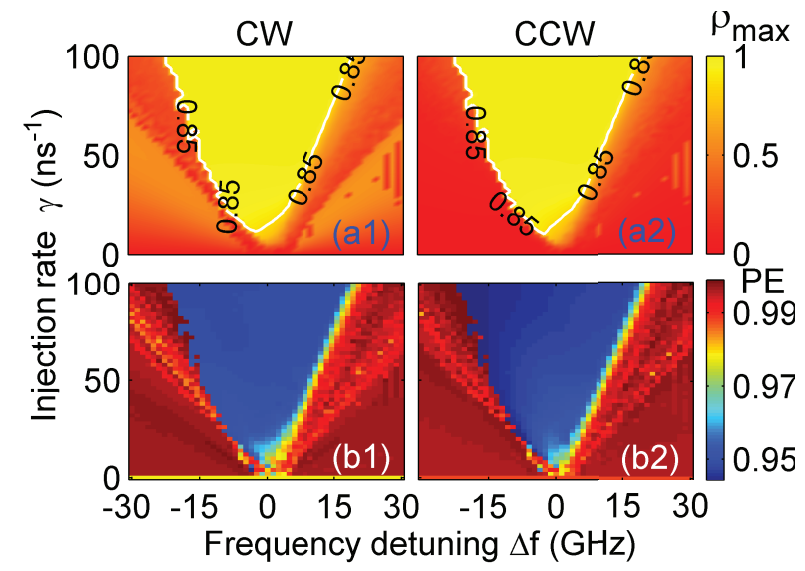

Fig. 10 (top) $\rho_{\max }$ between the two SRLs and (bottom) PE of the S-SRL as functions of $\gamma$ and $\Delta f$. Left: CW; right: CCW.

TD features [Fig. 9(a1-a2,b1-b2)]. The regions for the suppression of the TD signatures with respect to the two delays $\left(\tau_{1}\right.$ and $\left.\tau_{2}\right)$ are clearly overlapped meaning that the same sets of parameters can be used to generate signals with simultaneous TD concealment in both directions. As compared to Fig. 5, the range values in which $\tau_{1}$ and $\tau_{2}$ are simultaneously concealed is broad, and is extended over both positive and negative frequency detuning $\Delta f$. Interestingly, there also exists regions where the signatures of all time-delays including the total delay $(T)$ are very weak. We have verified that, in addition, none of the time-delays can be determined from the cross-correlation between the CW and CCW modes. The results in Fig. 9 reveal that the significant reduction of TD signatures can be achieved in a large parameter range from the S-SRL when it is injected by a M-SRL subject to COF configuration.

It has been found that the complexity of the slave laser can increase when the master and the slave are unlocked. This further complexity can be benefit for obtaining weak TD signatures $[18,26,27]$. In order to determine the similarity between the system complexity and the injection-locking, we show in Fig. 10 the peaksizes in the cross-correlation $\rho_{\max }$ and the system complexity characterized by permutation entropy (PE) [27-29]. To estimate PE, we choose the embedding dimension $D=5$ and the embedding delay $\tau_{e}=\tau_{1} \Omega_{s}$ (with $\Omega_{s}=1 \mathrm{ps}^{-1}$ being the sampling rate) for the sampled time series $[18,22]$. It is worth noting that the estimation of PE is very sensitive to the choice of the embedding delay, which accounts for the time scales of the underlying dynamics [29]. Again similar results are obtained for the CW and CCW modes. When comparing to Fig. 9, there is a clear overlap between the region where TD signatures are well reduced and the region where the complexity of the system is larger (higher $\mathrm{PE})$, outside the injection-locking region $\left(\rho_{\max }<0.85\right)$. This further confirms that the suppression of TD signatures and complexity enhancement are achieved, providing that the operation of the master-slave system stays away from injection locking. This has also been previously observed and explained for other optically injected laser systems $[16,18]$. Moreover, one can also notice the difference in complexity between the regions where all time-delays are effectively suppressed and where only the delay multiples can be identified [see Fig. 10(b)].

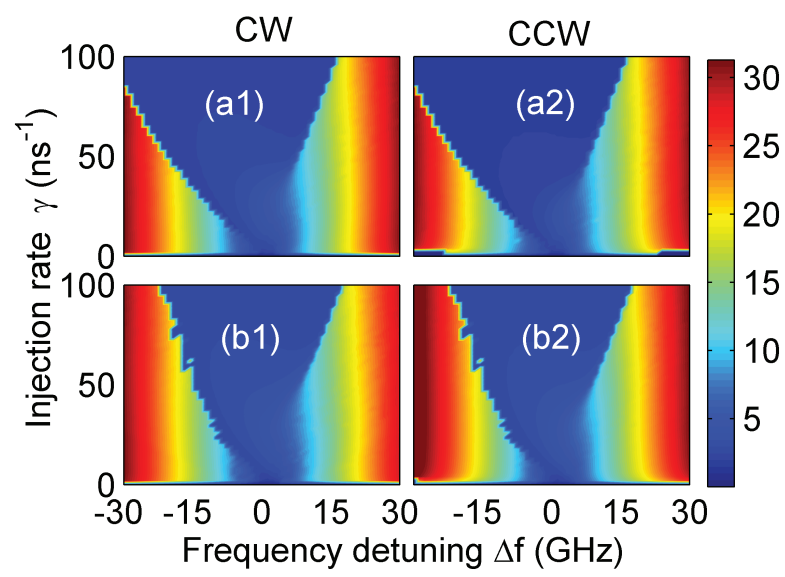

Fig. 11 Bandwidth of the S-SRL when its M-SRL under SOF (top) and COF (bottom). Left: CW; right: CCW. 

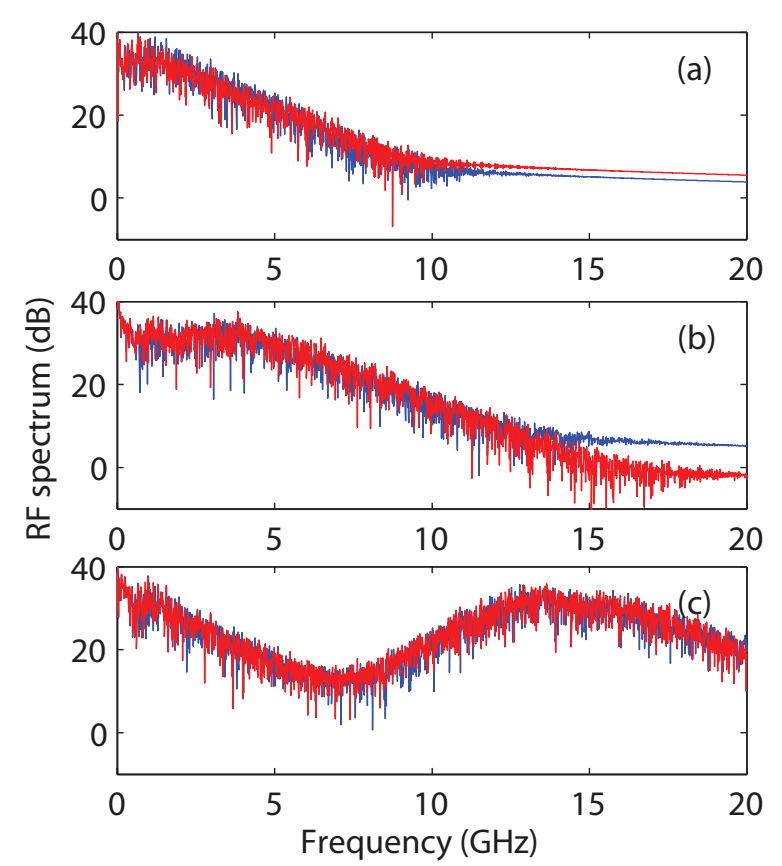

Fig. 12 Examples of rf spectrum: (a) the M-SRL under COF, (b) the S-SRL when the injection parameters are $\Delta f=5 \mathrm{GHz}$ and $\gamma=10 \mathrm{~ns}^{-1}$, and (c) the S-SRL when the injection parameters are $\Delta f=15 \mathrm{GHz}$ and $\gamma=48 \mathrm{~ns}^{-1}$.

\section{Bandwidth enhancement}

In the previous section, we have shown the concealment of TD signatures in an S-SRL optically injected by an M-SRL. In the parameter plane of injection rate and frequency detuning, an improvement in TD suppression and complexity has been found, especially when the M-SRL subject to COF. Now we explore whether enhanced bandwidths can be obtained in $(\Delta f$, $\gamma$ )-plane where TD concealment occurs. To this end, we define the value of the bandwidth as that for which the range between the DC and the frequency contains $80 \%$ of the spectral power. Figure 11 shows in the $(\Delta f, \gamma)$-plane of how the calculated bandwidths of the S-SRL evolve, for both the cases of the M-SRL under $\mathrm{SOF}$ and COF. The color maps clearly indicate that the chaos bandwidth of the S-SRL can be significantly enhanced when the injection parameters are chosen outside the injection-locking region. It is worth noting that the calculated bandwidths are very sensitive to the value of the frequency detuning. This is because there is a peak around the detuning in the radio-frequency (rf) spectrum which represents a frequency resulting from strong beating between the S-SRL field and the injected light from the M-SRL, as will be shown below. An example of bandwidth comparison between the M-SRL under COF and the S-SRL is presented in Fig. 12; Fig. 12(a) shows the rf spectrum of the M-SRL as reference.
As shown in Figs. 12(b) and (c), broader and more flat rf spectra are obtained in the S-SRL indicating that, for appreciate parameters, the S-SRL exhibits faster oscillations than the master. A careful comparison to Figs. 7(b) and (c) shows that chaos bandwidth enhancement and very good TD suppression are simultaneously realized, which represents a vivid example of enhanced chaotic dynamics generated in the S-SRL.

Table 1 Results of NIST statistical tests for bit sequences based on the M-SRL. The results have been performed using 1000 samples of 1 Mbit data. The significance level, for Success, i.e., the $P$ value ( $u-$ niformity of $p$ values) should be larger than 0.0001 and the proportion should be in the $0.99 \pm 0.0094392$ range [30]. For the tests that produce multiple $P$ values and proportions, the worst case is shown.

\begin{tabular}{lclc}
\hline \hline Statistical test & P value & Proportion & Result \\
\hline Frequency & 0.469232 & 0.9940 & Success \\
Block frequency & $\mathbf{0 . 0 1 9 9 9 3}$ & $\mathbf{0 . 9 7 8 0}$ & Failure \\
Cumulative sums & 0.765632 & 0.9930 & Success \\
Runs & 0.160805 & 0.9840 & Success \\
LongestRun & 0.994720 & 0.9910 & Success \\
Rank & 0.130369 & 0.9950 & Success \\
FFT & 0.919131 & 0.9870 & Success \\
NonOverlappingTemplate & 0.019188 & 0.9910 & Success \\
OverlappingTemplate & 0.382115 & 0.9830 & Success \\
Universal & 0.184549 & 0.9840 & Success \\
ApproximateEntropy & $\mathbf{0 . 0 0 2 7 7 8}$ & $\mathbf{0 . 9 7 6 0}$ & Failure \\
RandomExcursions & 0.001425 & 0.9902 & Success \\
RandomExcursionsVariant & 0.164844 & 0.9935 & Success \\
Serial & $\mathbf{0 . 0 0 0 0 6 4}$ & $\mathbf{0 . 9 5 2 0}$ & Failure \\
LinearComplexity & 0.284024 & 0.9860 & Success \\
\hline \hline
\end{tabular}

Table 2 Results of NIST statistical tests for bit sequences based on the S-SRL.

\begin{tabular}{lclc}
\hline \hline Statistical test & P value & Proportion & Result \\
\hline Frequency & 0.311542 & 0.9920 & Success \\
Block frequency & 0.846338 & 0.9920 & Success \\
Cumulative sums & 0.428095 & 0.9890 & Success \\
Runs & 0.151190 & 0.9940 & Success \\
LongestRun & 0.620465 & 0.9850 & Success \\
Rank & 0.618385 & 0.9900 & Success \\
FFT & 0.743915 & 0.9910 & Success \\
NonOverlappingTemplate & 0.010606 & 0.9860 & Success \\
OverlappingTemplate & 0.626709 & 0.9940 & Success \\
Universal & 0.167184 & 0.9840 & Success \\
ApproximateEntropy & 0.355364 & 0.9880 & Success \\
RandomExcursions & 0.053926 & 0.9889 & Success \\
RandomExcursionsVariant & 0.036868 & 0.9857 & Success \\
Serial & 0.316052 & 0.9910 & Success \\
LinearComplexity & 0.866097 & 0.9940 & Success \\
\hline \hline
\end{tabular}




\section{Potential Benefit of Improved Chaotic Dynamics}

In order to link the enhanced chaotic dynamics to the improvement in the performance of a potential application, we now compare the randomness properties of two bitstreams constructed from the master and slave output signals simultaneously recorded. Concretely, we apply the same post-processing to master signals and slave signals that are the response output signals of the slave to the same master signals. The parameters are the same as in Figs. 12(a) and (b), where the TD signature in the master signal is very obvious while that of the slave signal shows strongly attenuated TD signature and also an improved bandwidth. In order to achieve efficient generation of random bits, we adopt the highorder finite differences (HFD) procedure (see [5] and references therein), which is efficient to extract random bits from any source of randomness with nonsymmetric distribution. One benefit of this post-processing is that it is not necessary to operate under the optimal conditions regarding the chaotic dynamics and acquisition process. This is because the calculation of HFD reduces dependence on these properties. It is highly efficient to generate bit sequences of 1 Gbits for randomness testing with the help of this approach, which leaves out the time-consuming procedure of generating very long chaotic signals with Eqs. (1)-(5).

For the two sequences, we carried out the 30thorder finite differences of a sequence of the floatingpoint numbers were calculated. The choice of the order of 30 th is somewhat arbitrary, but this means that only about $4 \times 10^{7}$ digitized samples are needed for generating 1 Gbits sequences. We test the random properties of our sequences using the NIST tests (NIST Special Publication 800-22 [30]). We found that an extraction of 25 least significant bits (LSBs) from each sample for the S-SRL passes all the NIST statistical tests for randomness (see Table II), while three tests (block frequency, approximate entropy, and serial) are not passed for the case of the M-SRL (see Table I). The reason for extracting only $25 \mathrm{LSBs}$ is that strong correlations appear in the bit stream after the calculation of HFD and one should discard certain most significant bits to eliminate these correlations. When $28 \mathrm{LSBs}$ out of 30 are retained from each sample (the results of the NIST tests are not shown here for this case), only 5 out of the 15 NIST tests passed for the sequence from the M-SRL signal while up to 12 tests passed for the corresponding sequence from the S-SRL signal. These two examples evidence that the injection of SRLs, as for other types lasers, can allow to improve the chaotic features (reduction of TD signatures, improved band- widths, etc..) useful for random bit generation. We have also tested simpler post-processing, say, LSB selection and bitwise exclusive-or (XOR) operation as that used in [31], based on the shorter bit sequence (160 Mbits). Again, we found that it is much easier for the bit sequence from the S-SRL signal to pass the NIST tests than for that from the M-SRL signal (not shown here) confirming the way of enhancing chaotic dynamics of SRLs through chaotic optical injection.

\section{Conclusion}

We have numerically investigated the conditions under which the optical injection into an SRL is helpful for the suppression of the TD signatures in chaotic signals generated by an SRL subject to feedback. Although our system requires the use of two different SRLs, it offers some attractive benefits including better the suppression of the TD signatures and enhanced chaos bandwidth in a wide region of the injection parameters. More importantly, these features can be obtained even for moderate values of linewidth enhancement factors and also with typical values of self-and cross-saturation terms as compared to [23]. In this study, we have considered two different feedback configurations: self-feedback and cross-feedback. When the slave SRL is injected by a master SRL subject to self-feedback, similar results as those obtained with optically injected EEL laser [13] and injected vertical-cavity surface-emitting lasers are obtained. Only a narrow range of injection rates and the detunings can be used to reduce significantly the T$\mathrm{D}$ signatures in this configuration. When the slave SRL is injected by a master SRL subject to cross-feedback, there exists a large range of injection rates and, positive and negative, detunings for which the two main timedelays embedded in the model can be suppressed. In addition, we have identified ranges of these parameters for which all the time-delays, including the total timedelay can be effectively suppressed. These features are unique for an SRL subject to chaotic optical injection from another SRL under cross-feedback. Moreover, our numerical results have also shown that the injection is suitable to obtain more broader signals the slave SRLs. To appreciate the impact of our results, we have used the NIST tests to compare the randomness of the bit sequences constructed using the output signals of the master SRL and the slave SRL. It has been evidenced that, unlike the bitstreams constructed from the master SRL signals, those constructed from the slave SRL signals with improved chaotic features passed all the NIST tests. Thus, our results contribute to further improve the randomness properties of chaos-governing bit- 
streams and also to enhance the security in chaos-based communications.

Acknowledgements This work was supported in part by the Engineering and Physical Sciences Research Council (Grant NO. EP/M0242371/1), and in part by the Conseil Regional de la Région Grand Est. R.M.N acknowledges the support of the F.N.R.S. (Belgium)

\section{References}

1. Sciamanna, M., Shore, K. A.: Physics and applications of laser diode chaos. Nat. Photonics 9, 151-162 (2015).

2. Nguimdo, R. M., Colet, P., Larger, L., Pesquera, L.: Digital key for chaos communication performing time delay concealment. Phys. Rev. Lett. 107, 034103 (2011).

3. Rontani, D., Choi, D., Chang, C. Y., Locquet, A., Citrin, D. S.: Compressive sensing with optical chaos. Sci. Rep. 6, 35206 (2016).

4. Jiang, N. Xue, C., Lv, Y., Qiu, K.: Physically enhanced secure wavelength division multiplexing chaos communication using multimode semiconductor lasers. Nonlinear Dyn. 86, 1937-1949 (2016).

5. Deng, T., Xia, G. Q., Wu, Z. M.: Broadband chaos synchronization and communication based on mutually coupled VCSELs subject to a bandwidth-enhanced chaotic signal injection. Nonlinear Dyn. 76, 399-407 (2014).

6. Winebarger, J., Locquet, A., Citrin,D.S.: Breaking on/off phase-shift keying in optical chaos-based cryptosystems. Proc. of SPIE 7720, 772025 (2010).

7. Li, N., Kim, B., Chizhevsky, V. N., Locquet, A., Bloch, M., Citrin, D. S., Pan, W.: Two approaches for ultrafast random bit generation based on the chaotic dynamics of a semiconductor laser. Opt. Express 22, 6634-6646 (2014).

8. Rontani, D., Locquet, A., Sciamanna, M., Citrin, D. S.: Loss of time-delay signature in the chaotic output of a semiconductor laser with optical feedback. Opt. Lett. 32, 2960-2962 (2007).

9. Nguimdo, R. M., Soriano, M. C., Colet, P.: Role of the phase in the identification of delay time in semiconductor lasers with optical feedback. Opt. Lett. 36, 4332-4334 (2011).

10. Li, S., Liu, Q., Chan, S. C.: Distributed feedbacks for time-delay signature suppression of chaos generated from a semiconductor laser. IEEE Photonics J. 4, 1930-1935 (2012).

11. Wang, A., Wang, B., Wang, Y., Shore, K. A.: Optical heterodyne generation of high dimensional and broadband white chaos. IEEE J. Sel. Topics Quantum Electron. 21, 1800710 (2015).

12. Cheng, C., Chen, Y., Lin, F.: Chaos time delay signature suppression and bandwidth enhancement by electrical heterodyning. Opt. Express, 23, 2308-2319 (2015).

13. Li, N., Pan, W., Xiang, S., Yan, L., Luo, B., Zou, X., Zhang, L., Mu, P.: Photonic generation of wideband timedelay-signature-eliminated chaotic signals utilizing an optically injected semiconductor laser. IEEE J. Quantum Electron. 48, 1339-1345 (2012).

14. Xiang, S., Pan, W., Zhang, L., Wen, A., Shang, L., Zhang, H., Lin, L.: Phase-modulated dual-path feedback for time delay signature suppression from intensity and phase chaos in semiconductor laser. Opt. Commun. 324, 38-46 (2014).
15. Wu, J., Wu, Z., Xia, G., Feng, G.: Evolution of time delay signature of chaos generated in a mutually delay-coupled semiconductor lasers system. Opt. Express 20, 1741-1753 (2012).

16. Hong, Y., Quirce, A., Wang, B., Ji, S., Panajotov, K., Spencer, P. S.: Concealment of chaos time-delay signature in three-cascaded vertical-cavity surface-emitting lasers. IEEE J. Quantum Electron. 52, 2400508 (2016).

17. Virte, M., Panajotov, K., Thienpont, H., Sciamanna, M.: Deterministic polarization chaos from a laser diode. Nat. Photonics, 7, 60-65 (2013).

18. Li, N., Pan, W., Locquet, A., Citrin, D. S.: Time-delay concealment and complexity enhancement of an externalcavity laser through optical injection. Opt. Lett. 40, 4416-4419 (2015).

19. Gao, X., Cheng, M., Deng, L., Liu, L., Hu, H., Liu, D.: A novel chaotic system with suppressed time-delay signature based on multiple electro-optic nonlinear loops. Nonlinear Dyn. 82, 611-617 (2015).

20. Ermakov, I. V., Van der Sande, G., Danckaert, J.: Semiconductor ring laser subject to delayed optical feedback: bifurcations and stability. Commun. Nonlinear Sci. Numer. Simul. 17, 4767-4779 (2012).

21. Sorel, M., Giuliani, G., Sciré, A., Miglierina, R., Donati, S., Laybourn, P. J. R.: Operating regimes of GaAsAlGaAs semiconductor ring lasers: experimental and model. IEEE J. Quantum Electron. 39, 1187-1195 (2003).

22. Nguimdo, R. M., Verschaffelt, G., Danckaert, J., van der Sande, G.: Delay signature concealment in chaotic semiconductor ring lasers. Proc. of SPIE, 9134, 913424 (2014).

23. Nguimdo, R. M., Verschaffelt, G., Danckaert, J., van der Sande, G.: Loss of time-delay signature in chaotic semiconductor ring lasers. Opt. Lett. 37, 2541-2543 (2012).

24. Nguimdo, R. M., Verschaffelt, G., Danckaert, J., Van der Sande, G.: Simultaneous computation of two independent tasks using reservoir computing based on a single photonic nonlinear node with optical feedback. IEEE Trans. Neural Netw. Learn. Syst. textbf26, 3301-3307 (2015).

25. Mashal, L., van der Sande, G., Gelens, L., Danckaert, J., Verschaffelt, G.: Square-wave oscillations in semiconductor ring lasers with delayed optical feedback. Opt. Express 20, 22503-22516 (2012).

26. Heiligenthal, S., Jüngling, T., D'Huys, O., ArroyoAlmanza, D. A., Soriano, M. C., Fischer, I., Kanter, I., Kinzel, W.: Strong and weak chaos in networks of semiconductor lasers with time-delayed couplings. Phys. Rev. E 88, 012902 (2013).

27. Toomey, J. P., Kane, D. M.: Mapping the dynamic complexity of a semiconductor laser with optical feedback using permutation entropy. Opt. Express 22, 1713-1725, 2014.

28. Bandt C., Pompe, B.: Permutation entropy: a natural complexity measure for time series. Phys. Rev. Lett. 88, $174102(2002)$.

29. Li, N., Zunino, L., Locquet, A., Kim, B., Choi, D., Pan, W., Citrin, D. S.: Multiscale ordinal symbolic analysis of the Lang-Kobayashi model for external-cavity semiconductor lasers: A test of theory. IEEE J. Quantum Electron. 51, 2200206 (2015).

30. Rukhin, A., Soto, J., Nechvatal, J., Smid, M., Barker, E., Leigh, S., Levenson, M.: Nat. Inst. Standards and Technology, Special Publication 80022, 2001, Revision 1a, 2010 [Online]. Available: http://csrc.nist.gov/publications/nist-pubs/800-22rev1/SP800-22rev1.pdf. 
31. Nguimdo, R. M., Verschaffelt, G., Danckaert, J., Leijtens, X., Bolk, J., Van der Sande, G.: Fast random bits generation based on a single chaotic semiconductor ring laser. Opt. Express, 20, 28603-28613 (2012). 